\title{
New Method in Calculating the Trajectory of Sound Waves at Stratified Ocean
}

\author{
V. P. Ivanov, G. K. Ivanova \\ Institute of Applied Physics, Russian Academy of Sciences, Nizhny Novgorod, Russia \\ Email:ivg@hydro.appl.sci-nnov.ru
}

Received 17 March 2016; accepted 27 June 2016; published 30 June 2016

Copyright (C) 2016 by authors and Scientific Research Publishing Inc.

This work is licensed under the Creative Commons Attribution International License (CC BY).

http://creativecommons.org/licenses/by/4.0/

(c) (i) Open Access

\section{Abstract}

There is a new method of calculating the trajectory of sound waves (rays) in layered stratified speed of sound in ocean without dispersion. A sound wave in the fluid is considered as a vector. The amplitudes occurring at the boundary layers of the reflected and refracted waves are calculated according to the law of addition of vectors and using the law of conservation of energy, as well as the laws that determine the angles of reflection and refraction. It is shown that in calculating the trajectories, the reflected wave must be taken into account. The reflecting wave's value may be about 1 at certain angles of the initial wave output from the sours. Reflecting wave forms the so-called water rays, which do not touch the bottom and the surface of the ocean. The conditions of occurrence of the water rays are following. The sum of the angles of the incident and refracted waves (rays) should be a right angle, and the tangent of the angle of inclination of the incident wave is equal to the refractive index. Under these conditions, the refracted wave amplitude vanishes. All sound energy is converted into the reflected beam, and total internal reflection occurs. In this paper, the calculation of the amplitudes and beam trajectories is conducted for the canonical type of waveguide, in which the speed of sound is asymmetric parabola. The sound source is placed at the depth of the center of the parabola. Total internal reflection occurs in a narrow range of angles of exit beams from the source $43^{\circ}-45^{\circ}$. Within this range of angles, the water rays form and not touch the bottom and surface of ocean. Outside this range, the bulk of the beam spreads, touching the bottom and the surface of the ocean. When exit corners, equal and greater than $77^{\circ}$, at some distance the beam becomes horizontal and extends along the layer, without leaving it. Calculation of the wave amplitudes excludes absorption factor. Note that the formula for amplitudes of the sound waves applies to light waves.

\section{Keywords}

Sound Wave, Rays, Water Ray, Trajectory, Liquid Space 


\section{Introduction}

The work is devoted to the study of the propagation of sound waves in inhomogeneous media, the speed of sound in which smoothly changes with depth. This environment may be modeled with a plurality of horizontal layers of constant speed of sound in each layer. This type of consideration depending on the depth of the sound speed is widely used in acoustics. A sound wave is described by a traveling wave phase of which consists of two components, depending on the spatial and temporal coordinates.

The direction of wave propagation in the majority of the work is determined by the wave phase. This is wrong. The wave phase does not contain its direction, as shown in [1]-[3]. In [4], there is proposed to consider the amplitude of the sound wave as vector having the direction of the trajectory of its propagation. The direction of the waves creates a sound source. A sound wave propagating in the liquid produces a pressure on the liquid, causing the deformation of the liquid. Pressure by definition is a vector having the same direction as that of the sound wave. The property of liquid is such that during propagation in the homogeneous medium the wave direction and pressure at fluid do not change.

It is known that at the boundary between two media of the incident wave arises two waves, reflection and refraction. The newly arisen wave also put pressure on the liquid. In [4], it is shown that the equality pressure projections reflected and refracted waves at the boundary of the medium is none other than the Snell's law, which determines the direction of propagation of refracted wave. In most studies, for example, [5], the pressure is considered as a scalar quantity. It uses to calculate coefficients of reflection $V$ and refraction $W$. One of the conditions of calculation $V$ and $W$ in [5] is following: there is equality in the sum of the incident and reflected pressure waves on one side and refracted wave on the other side of the boundary. This condition is wrong, because the incident wave is counted twice, both above and below the boundaries of media-in refracted wave. In addition, it is unnecessary, because the pressures of reflected and refracted waves are equal to each other according to Snell's law, which determines the direction of propagation of refracted waves. In [4], to calculate the amplitudes of the waves arising at the interface, vector addition of the amplitudes of the waves is used, similar to the law of conservation of momentum in the mechanics and the law of conservation of energy.

In the future, for the wave passing through the boundary, instead of the term "refracted", it will be used the term "passing", leaving the term "refracted" to refer to beam angle, passing through the boundary of the space.

The purpose of this work is to calculate the trajectories of sound rays, using the formula for amplitudes of reflected and passing waves, received in [4].

\section{Calculating Trajectories of Wave Propagation in an Inhomogeneous Space Based on the Reflected and Transmitted Waves}

We assume that the sound wave propagation occurs in an not uniform space where the speed of sound varies continuously in depth, while remaining constant in the layer thickness $\delta z$ equal to the speed of sound at a depth of layer. The amplitudes of the reflected and passed waves at the boundaries between the layers of the liquid with constant speed of sound in each layer determined by the following formulas [4]:

$$
V=\frac{1-n^{2}}{\sqrt{4 n^{2} \cos ^{2}\left(\theta+\theta_{1}\right)+\left(1-n^{2}\right)^{2}}}, W=\frac{2 n \cos \left(\vartheta+\theta_{1}\right)}{\sqrt{4 n^{2} \cos ^{2}\left(\theta+\theta_{1}\right)+\left(1-n^{2}\right)^{2}}} .
$$

Here $V, W$-modules amplitude of the reflected wave and the wave passing through the boundary between the layers to the next layer. These formulas are applied at the boundaries of layers in depth, at which the waveguide divide. The value $n=c / c_{1}$-refractive index at the interface of adjacent layers, $c$ - sound velocity in the layer where the initial wave comes, $c_{1}$-followed next layer. The angles $\theta$ and $\theta_{1}$ of incident and passed waves took place in the neighboring layer waves, measured from the vertical axis. Formula (1) applies for any value of the coefficient $n$, greater than or less than 1 , it is easy to show by replacing in the formulas (1) $n$ for $n^{\prime}=1 / n$.

It can be seen that the amplitude of the reflected wave $V$ as a function of $n$ is less than the amplitude of the transmitted wave $W$ and vanishes only in a homogeneous medium with $n=1$. The amplitude of the transmitted wave $W$ depends on the sum of the angles $\theta$ and $\theta_{1}$, by which the waves propagate in the adjacent layers. If $\theta+$ $\theta_{1}=\pi / 2$, then $V=1$ and $W=0$. This means that the transmitted wave $W$ is absent, its amplitude is zero. As a result the incident on the boundary layer wave is reflected totally with the opposite sign of the vertical component of the vector $\boldsymbol{V}$ at an angle $\theta$ falling. This process is called total internal reflection (TIR). When TIR arise sound 
wave with the changed direction enters the layer, from which it came, and already is the incident wave with respect to the adjacent layer. At the boundary of the next layer there form two waves: transmitted wave $W$ and the reflected wave of the $V$, directed towards the layer, where the transmitted wave disappeared. There has been a change in direction of propagation of the wave without contact with the bottom or surface. The water wave (ray) appears. From (1) it follows that the formation of water rays in inhomogeneous media can only occur when the corners of the output rays from a source less than $45^{\circ}$. As a result of refraction the sum of angles the sum of $\theta+$ $\theta_{1}$ increases, when it reaches $90^{\circ}$ TIR comes. It follows that in calculating the trajectories of waves propagating in not uniform environment, you must take into account both waves, the reflected and transmitted. At certain output angles, and the value of the refractive index, they can pass one another without touching the bottom and surface forming a water rays in the waveguide.

Thus, the TIR process, the change of direction of propagation of the beam about depth, both above and below the axis of the waveguide, leads to the formation of water ray cycles without touching the surface of the waveguide and a bottom.

It is known that $\cos \varphi$, while $\varphi \approx \pi / 2$ is changing quickly enough in comparison with the region where the phase $\varphi$ is close to zero. Therefore, the change in direction of the sound wave amplitude at TIR takes place in the space of $\delta x, \delta z$ small extent.

At present, the trajectories calculation is carried out without regard to the reflected wave, [5], considering that its amplitude $V$ at small gradients of sound speed in waveguides is much smaller than the amplitude of the transmitted wave $W$. However, it is assumed that water rays, that not touch the bottom and the surface, exist. In [5] there is adopted the following mechanism for the formation of water rays. During propagation resulting refraction angle between the beam and axis $z$, reaches a value of $90^{\circ}$ and ray becomes horizontal, then ray turn to the opposite direction. In the literature, the beam turning is not explained, but simply postulated. Consider the possibility of change of the vertical component of the unit vector along the trajectory of the beam on the contrary that is assumed in [5].

The difference between the angles $\theta, \theta_{1}$ is small, $\theta_{1}=\theta+\delta \theta$. Let us assume that $\theta_{1}$-angle of refraction is equal to $90^{\circ}$. According to Snell's law, $\sin \theta=n \sin \left(\theta_{1}\right)=\sin (\theta+\delta \theta)=n$, the refracted beam is horizontal. For horizontal beam trajectory is an infinite horizontal line at the depth where the angle of refraction become equal to $90^{\circ}$. There is no way to stop this line and turn in the desired direction. Where the beam becomes horizontal, the trajectory becomes infinite. In formula (1) $W$ consists of angles incident on the layer, and transmitted waves: $\cos$ $\left(\theta+\theta_{1}\right)=\cos (\theta+\theta+\delta \theta)=\cos (180-\delta \theta)=-\cos (\delta \theta)$-finite value. According to formulas (1) it is possible to calculate the amplitude of the transmitted and reflected beams for the different propagation angles.

To calculate the trajectories adopted canonical type waveguide [6]. Sound velocity distribution there is an asymmetric parabola. Center of the parabola is placed at a depth of $1 \mathrm{~km}$, depth waveguide is $4 \mathrm{~km}$, thickness of each layer is $25 \mathrm{~cm}$. The amplitudes of the rays at the boundary layers are calculated by the above formulas (1).

Assume the following boundary conditions for $V$ and $W$ : when the sound wave touches the ocean surface and the bottom there is a full reflection of the waves. Upon reflection, according to [1]-[3], the wave phase $\varphi=k R$ remains unchanged since reflection occurs at one point. Vector modules $k, R$ does not change. Figure 1 shows the dependence of the sound velocity in $z$ depth of the waveguide.

We assume that the sound source emits a spherical wave. The refractive indices $n_{0}=c_{1} / c_{0}=0.9762, n_{4}=c_{1} / c_{4}$ $=0.9942, c_{1}$-speed at the depth of source $z_{1}=1 \mathrm{~km}, c_{0}, c_{4}$-at the surface and bottom. During the propagation the beam from source to the bottom or to surface refraction occurs, and its trajectory is deflected towards the source location of the horizon, $z_{1}=1 \mathrm{~km}$. Using the law of refraction there is can be calculated, at which the output angle the beam becomes horizontal: $\sin \theta=n \sin \theta_{1}$. When $\theta_{1}=90^{\circ} \sin \theta=n, \theta \approx 77^{\circ} \div 84^{\circ}$.

The trajectory of the sound beam is based on the coordinates of the beam changes from layer to layer. We introduce the following notation: $i$-layer number, sign $(+)$ means that the wave propagates in the direction of the bottom, (-) in the direction of the surface, $z_{i}=z_{0} \pm s t z * \mathrm{i}, x_{i}=x_{o}+\operatorname{stz} * \operatorname{tg}\left(\theta_{i}\right), x_{0}=0, z_{0}=1 \mathrm{~km}$-initial coordinates of the beam at the exit of the source, $\theta_{i}$-angle of beam spread in a layer $i$, stz $=0.25 \mathrm{~m}$-thickness of each layer along the $z$ axis.

Figure 2 shows the trajectory of the sound ray emerging from the source toward the bottom at an angle $\theta=$ $44^{\circ}$, which is measured from the $\mathrm{z}$ axis. The trajectory looks like a broken curve. Trajectory seems as straight line, despite the fact that there is refraction, but the trajectory curvature scale pattern invisible. The first break point of the trajectory, $\mathrm{x} \approx 2 \mathrm{~km}, \mathrm{z} \approx 3 \mathrm{~km}$, there is TIR, the transfer of all the sound energy to the reflected beam. Indeed, at this point the sum of the angles $\theta+\theta_{1}$ increase at $2^{\circ}$ and reach $90^{\circ}$. There was TIR and, according to 


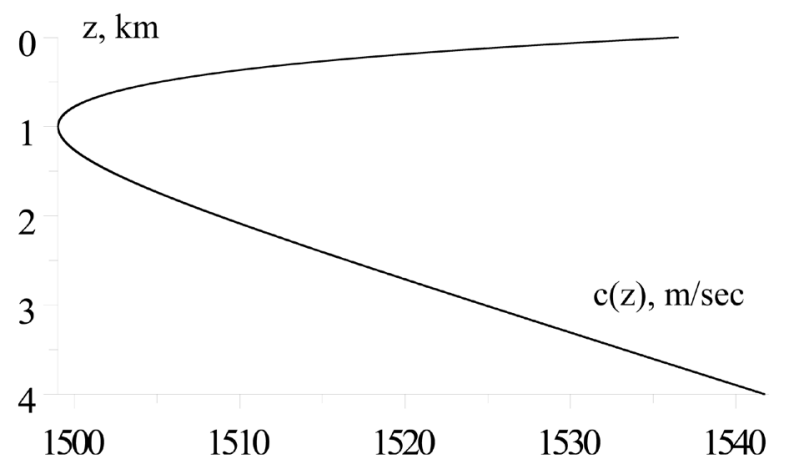

Figure 1. Dependence the sound velocity c(z) of the depth of the waveguide.

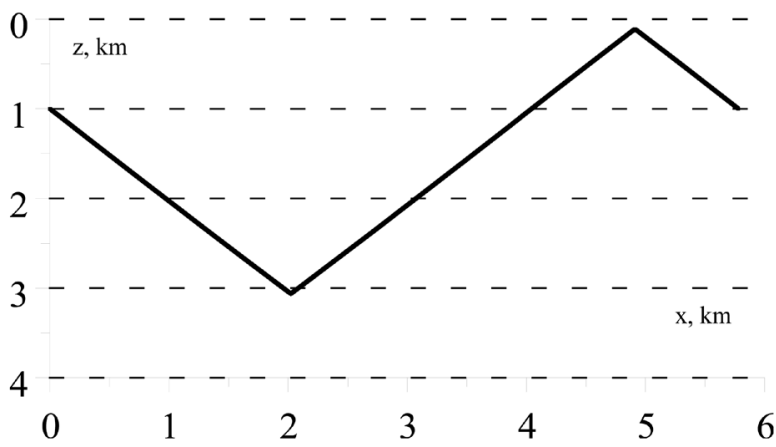

Figure 2. Trajectory of the sound beam, the exit angle $\theta=44^{\circ}$.

the formulas (1), the amplitudes $W=0, V=1$. The direction of propagation of sound turn in depth to the contrary, the beam turns towards the surface. In the second break point $x \approx 5 \mathrm{~km}, z \approx 0.111 \mathrm{~km}$ near the surface occurs again TIR: the sum of the angles $\theta+\theta_{1}$ also is $90^{\circ}, W=0, V=1$. Energy transmitted wave transmit reflected wave directed toward the bottom. After the second TIR beam returns to the source at the horizon of source, $z$ $=1 \mathrm{~km}$, completed the first full cycle of its propagation in the waveguide. Cycle length $\mathrm{D} \approx 6 \mathrm{~km}$, thus, a water ray (wave) appear and not touching the bottom and the surface of the waveguide.

Figure 3 shows how change the angles $\theta$ and $\theta_{1}$ along the trajectory shown in Figure 2, as a result of refraction. Two curves $\theta(x, z)$, and $\theta_{1}(x, z)$ in Figure 3 as a function of the distance $\mathrm{x}$ from the source merged into a single curve, because change the speed of sound between adjacent layers and causes a little small on the scale of the drawing differences and angles of propagation $\theta$ and $\theta_{1}$. Angles are both the functions of depth.

Figure 3 shows how change the angles $\theta$ and $\theta_{1}$ along the trajectory shown in Figure 2, as a result of refraction. Two curves $\theta(x, z)$, and $\theta_{1}(x, z)$ in Figure 3 as a function of the distance $x$ from the source merged into a single curve, because change the speed of sound between adjacent layers a little small and causes in the scale of the drawing small differences angles of propagation $\theta$ and $\theta_{1}$. Angles are both the functions of depth.

When the waves propagate the angle changes $\theta, \theta_{1}$ accumulate. The angle of inclination of the trajectory when ray exit from the source to the bottom increases from $44^{\circ}$ at the horizon of the source $\left(z_{0}=1 \mathrm{~km}\right)$, to the $45^{\circ}$ at break point of trajectory with $z \approx 3 \mathrm{~km}, x \approx 2 \mathrm{~km}$, forming first point TIR. Reflected at this point the wave propagates in the direction to the surface of the waveguide, its angle decreases (Figure 2) and at the horizon of the source becomes equal to $44^{\circ}$, forming a point of inflection. Then, the angle increases again, as a result of refraction and reaches $45^{\circ}$ at coordinate $x \approx 5 \mathrm{~km}$ and $z \approx 0.1 \mathrm{~km}$. There comes a new TIR wave near the surface. Figure 2 and Figure 3 show how the beam is formed D cycle length $\approx 6 \mathrm{~km}$. Throughout the cycle, the beam does not touch the bottom and the surface and twice change the direction of the velocity component along the $z$-axis. Water beam propagates in the depths of $0.1 \div 3 \mathrm{~km}$.

Figure 4 shows how change along the horizontal distance $x$ axis $V$ and $W$ amplitudes of the reflected and transmitted waves at an angle out of the source $44^{\circ}$. The red line shows the modulus of the amplitude of the transmitted wave $W$, black-of the reflected wave $V$. The vertical lines-wave amplitudes $V, W$ at the points of turn up (TIR) at $x=2$ and $5 \mathrm{~km}$. There are the points of direction change trajectory in Figure 2. The amplitude of the 


$$
\mathrm{z}=3 \mathrm{~km} \quad \mathrm{z}=011 \mathrm{~km}
$$

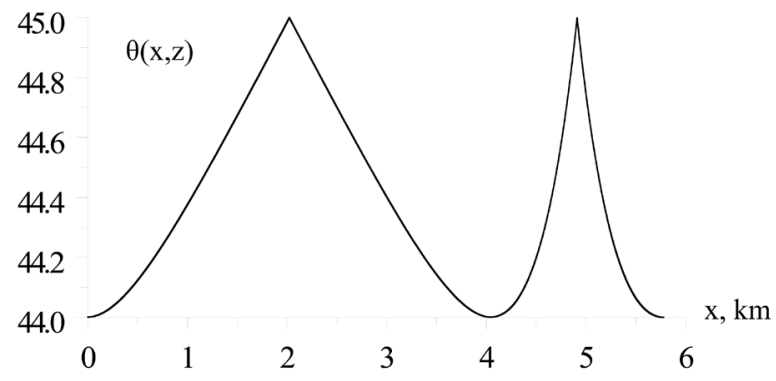

Figure 3. Changing the angles $\theta$ and $\theta_{1}$ along one cycle D.

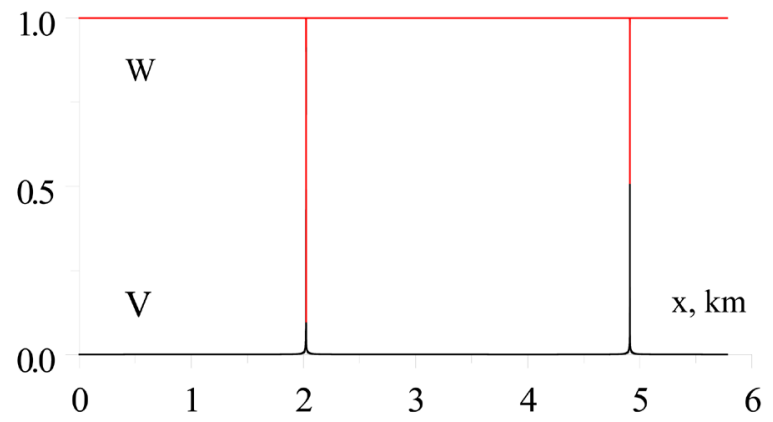

Figure 4. Modules $V$ the amplitude of the reflected wave and the passed $W$ as $x$ function, $\theta=44^{\circ}$.

reflected wave $V$ between the points of TIR is so small that in Figure 4 merges with the axis of $x$. The scale of Figure 2, 4 transfer of sound energy from the passing waves to the reflected visually occurs at one point $x$ axis. The area of TIR, the transition energy of the transmitted wave to the reflected and from reflected to the transmitted shown in Figure 5.

Pay attention to the following. The value of the amplitude of the transmitted wave (red line) is close to the maximum value, unit, all along the path with the exception of TIR region. As shown in Figure 5, the width of the field of TIR $\approx 10 \mathrm{~m}$ along the $\mathrm{x}$-axis close to $2 \mathrm{~km}$ and somewhat less close $x \approx 5 \mathrm{~km}$. TIR area range in the $z$ axis $V$ of the order of 10 benchmarks, $\delta z_{v}=$ stz $* 10=2.5 \mathrm{~m}$, for $W$-order 5 points, $\delta z_{w}=1.3 \mathrm{~m}$. In TIR area transmitted wave $W$ can be smaller than 1 and equal zero. The amplitude of the reflected wave $V$ outside of TIR is several orders of magnitude less 1 , but never vanishes. At TIR points amplitude $V$ is close to 1 . The above calculation shows that take account of the reflected wave in the construction of trajectories is necessary. Without its accounting reversed beam direction and the formation of water rays cannot be recorded.

The lowest water exit angle of rays in the waveguide is $\theta \approx 43^{\circ}$. For smaller angles of exit rays reflect from the surface and bottom of the waveguide. Figure 6 shows the beam path to the exit angle of $43.5^{\circ}$.

The first break point of the curve is near the bottom, at a depth of $z=3.888 \mathrm{~km}$ and does not touch the bottom. The second break point touches the surface.

Figure 7 shows the amplitude of the reflected and transmitted waves, $V$-black line, $W$-red line.

As it follows from Figure 6, at this go out angle there is only one output TIR point near the bottom. At a distance of $\approx 3 \mathrm{~km}$ beam turned toward the surface, and without experiencing the second TIR, came to the surface, reflected from it, and then went down to the level of the output from the source, $z_{1}=1 \mathrm{~km}$. The cycle of this beam is finished.

Figure 8 shows the amplitude of the reflected and transmitted waves, $V, W$, in the TIR in Figure 7 on an enlarged scale. They differ a little from those in Figure 5 to exit angle $44^{\circ}$.

Obviously, at lower exit angles rays touch the bottom and the surface.

It is shown that at an angle $\theta=45^{\circ}$ output rays touch the bottom and the surface, the point of TIR absent. With further increase in the angle of exit rays are retained by the bottom and surface up to angles $\theta \approx 75^{\circ}$. Figure 9 shows the trajectory of this ray. 

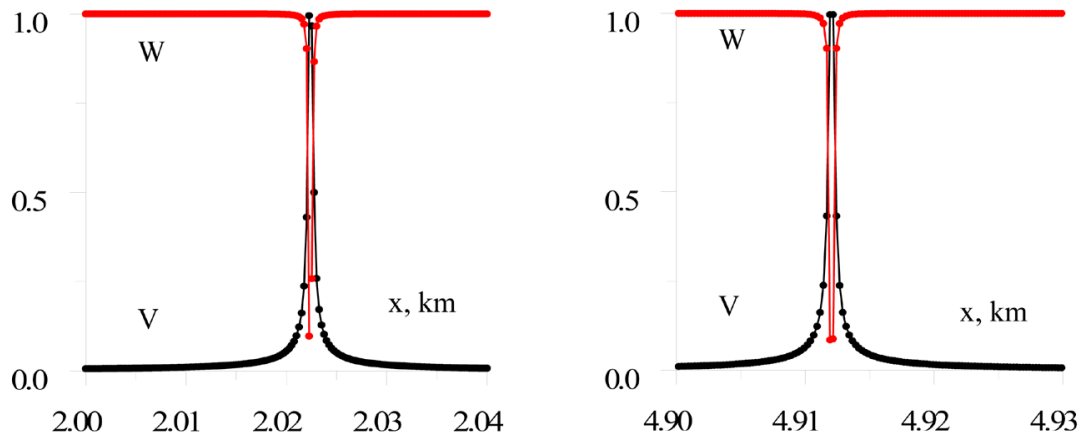

Figure 5. The amplitudes of $V$ and $W$ rays in the inversion points $=44^{\circ}$.

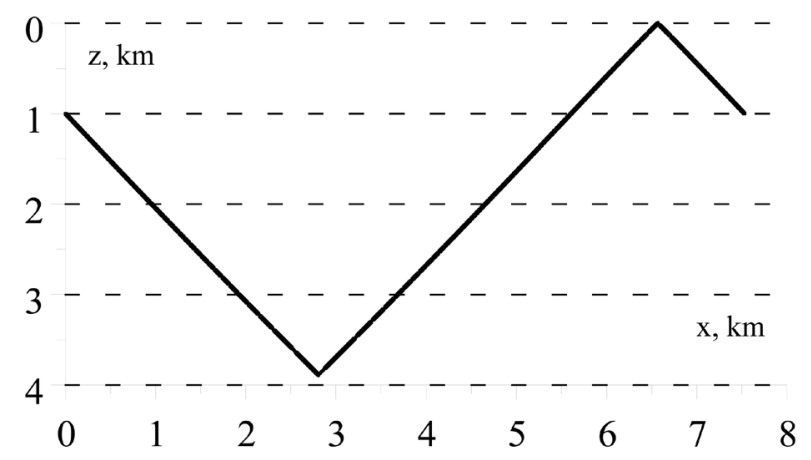

Figure 6. The trajectory of the beam output angle $\theta=43.5^{\circ}$.

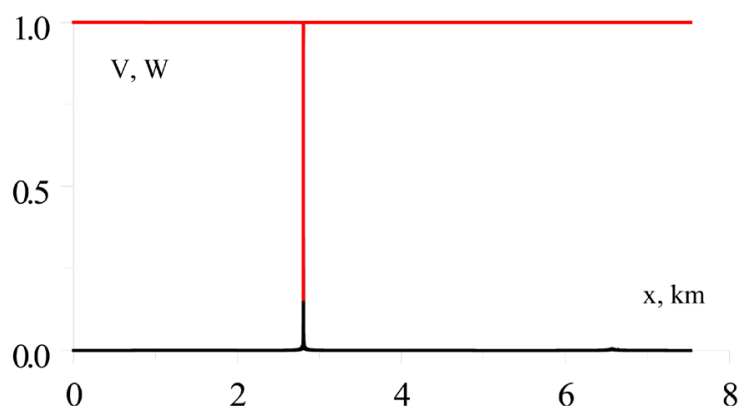

Figure 7. The amplitudes of the reflected and transmitted waves, $\theta=43.5^{\circ}$.

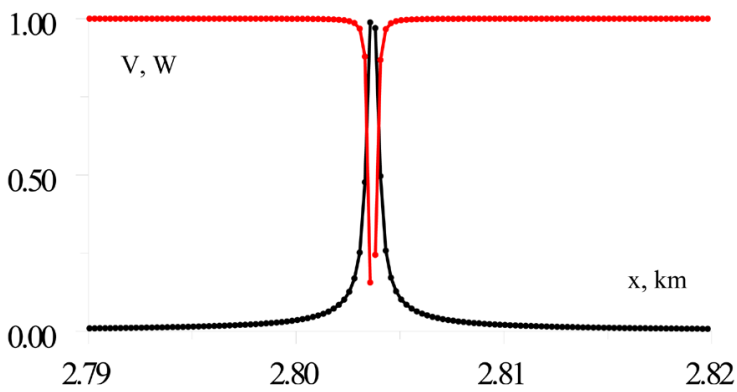

Figure 8. Scope of twisting beam output angle $\theta=43.5^{\circ}$.

At this beam output angle the curvature of the trajectory can be seen as a result of refraction and on the horizon the source $z=1 \mathrm{~km}$ noticeable inflection curve. Figure 10 and Figure 11 show the trajectories at exit angles $\theta \approx 77^{\circ}$ and $80^{\circ}$. 


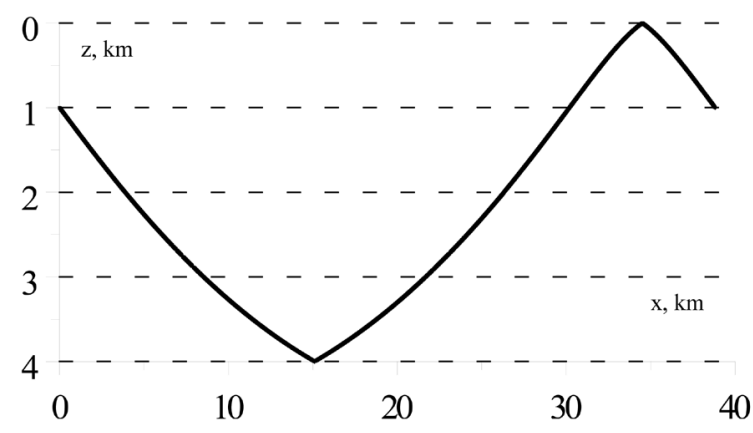

Figure 9. The path of the beam with an angle $\theta=75^{\circ}$ exit.

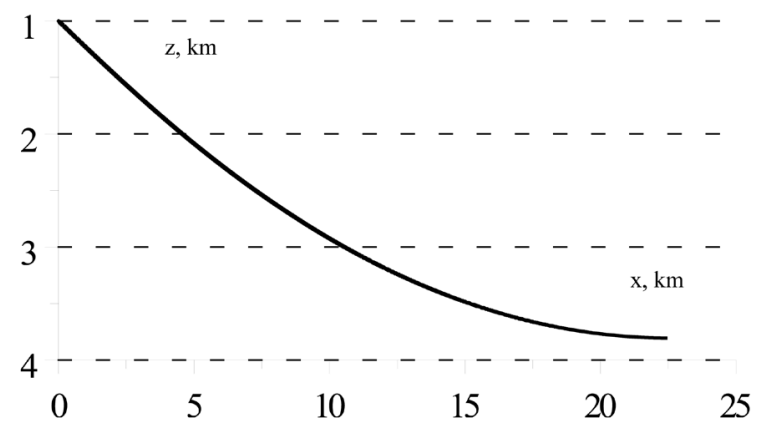

Figure 10. The path of the beam at $\theta=77^{\circ}$.

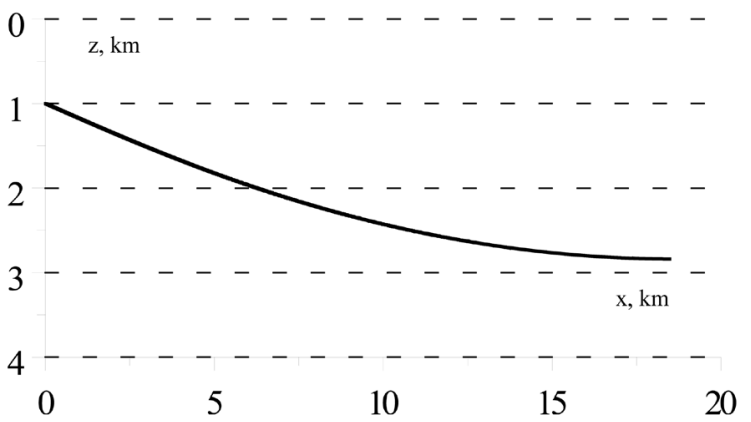

Figure 11. The trajectory of the beam at $\theta=80^{\circ}$.

Figure 10 and Figure 11 show that when exit angles greater than $77^{\circ}$, the beams do not touch the bottom and the surface and at some distance become near to horizontal. The following is a calculation of the output beam angle for the refractive index close to the bottom and the surface, when the rays are close to horizontal, $\theta_{1}=90^{\circ}$ : $\sin \theta=n=0.9762, \theta=77^{\circ} 30^{\prime}, \sin \theta=n=0.9942, \theta=83^{\circ} 50^{\prime}$.

\section{On the General Properties of Wave Processes of Different Physical Nature}

The formulas for the reflection coefficient (CR) and passing (CP) obtained in [5] and other works for the acoustic waves in liquid media coincide with similar formulas for light waves, Fresnel formulas [7]. The only difference is that in the formula include the acoustic density ratio adjacent media. It is usually close to unity. It turns out that the wave completely different physical nature, the longitudinal acoustic waves and transverse electromagnetic (EM) waves of light, on the border of homogeneous media have the same formulas for the reflection and transmission coefficients. This is not surprising. They are obtained at the same boundary conditions, which involve only the trajectory direction of waves and propagation speeds.

In optics, the light wave is created atoms of matter that emit light waves of different polarization and with different initial phases. It is known to observe the interference in natural light, a special device is used, for ex- 
ample, Fresnel mirrors [8], which is divided the light beam into two beams and keep its structure unchanged. This suggests that the polarization of the light wave and its initial phase is stable and does not depend on the propagation of the light wave.

It is clear that the CR and the CP for any wave must not depend on the phase and polarization at the boundary of spaces. To eliminate wave's phases of the processes at the boundary in [1]-[3], it is assumed that the initial phase of the reflected and transmitted waves equals to the phase of the incident wave. At the point of occurrence of the incident wave phase change does not occur, the modules of the vectors $\mathrm{k}$, $\mathrm{R}$ do not change. At the boundary the phase change did not happen, newly arisen waves kept the coherence between the incident wave and each other. At the intersection in space caused waves can interfere with each other, their time course is not broken, the difference between the phases of the waves depends on the distance covered after the occurrence of the way in a new environment.

Effect of polarization of light can also be excluded as the influence of phase waves at the point of reflection and refraction at the interface. We assume that during the formation of the incident wave reflected and transmitted all the characteristics of the original wave, including the polarization of the incident wave and its phase is passed unchanged the newly formed waves. If we take the boundary conditions are the same as in [4], we obtain formula (1) for light waves.

These findings can be confirmed by calculation. The paper [7] projection amplitudes of the vectors $\mathbf{E}$ and $\mathbf{H}$ on the axis of coordinates for the reflected and refracted waves at the interface expressed in terms of the projections of the vectors $\mathbf{E}$ and $\mathbf{H}$ of the original wave. Using the same method for calculating wave amplitude occurred and the boundary conditions are the same as in [4], we obtain formula (1) and for the light waves.

\section{Conclusions}

Trajectories of sound waves make using a new approach to the description of sound propagation in inhomogeneous media in depth.

It is assumed that the amplitude of the sound wave is a vector whose direction is given by the sound source. The pressure produced by the sound wave in the liquid is a vector too parallel to the direction of wave propagation.

To calculate the amplitude of the reflected and transmitted waves, vector summation of amplitudes of the waves is used, which is similar to the law of conservation of momentum of material particles. The second condition for calculating the amplitudes of the waves is the law of conservation of energy.

It was found that there exist water rays that not touch the bottom and the surface of the medium under certain conditions. They are the results of total internal reflection of the wave, when all the sound energy is converted to the reflected beam and the amplitude of the transmitted wave vanishes.

When total internal reflection axis, a sign of the vertical component of the amplitude of the reflected wave, is opposite to the wave incident on the interface, the direction of wave propagation along the vertical is reversed, it remains the same along the horizontally.

Water rays occur when the sum of the angles of the incident and transmitted waves is $90^{\circ}$, and the tangent of the angle of inclination of the incident wave is equal to the refractive index.

The range of output angles of rays from a source in which there are water rays does not exceed $45^{\circ}$. The lower boundary of occurrence of water rays in the waveguide is considered the angle of $\approx 43^{\circ}$.

When exit angles equal to $45^{\circ}$ and later $77^{\circ}$ in this waveguide, rays reflect from the bottom and the surface. At large angles of rays of output as a result of refraction, rays not close to the surface or the bottom, and become horizontal and distributed in a liquid made on the horizon, without changing its direction of propagation.

After the elimination of errors in the understanding of the scale production of vectors $\mathbf{k}, \mathbf{R}$, calculation of wave amplitudes can be made at any depth of the waveguide.

It is assumed that the phase of the incident at the boundary of a sound wave is the initial phase of the reflected and transmitted waves. This leads to the continuous of phases of the arising waves and their coherence.

\section{References}

[1] Ivanov, V.P. and Ivanova, G.K. (2013) A New Concept of Calculation Coefficients of Reflection Passage Sound Waves on the Boundary of Liquid Spaces. Proceedings of the 14th L.M. Brekhovskikh Conference, Moscow, 121-124.

[2] Ivanov, V.P. and Ivanova, G.K. (2013) A New Concept of Calculation Coefficients of Reflection Passage Sound 
Waves on the Boundary of Liquid Spaces. OJA, 3, 72-79. http://dx.doi.org/10.4236/oja.2013.33012

[3] Smirnov, V.I. (1958) A Course of Higher Mathematics.

[4] Ivanov, V.P. and Ivanova, G.K. (2015) To the Question of Sound Waves Propagation in Liquids. OJA, 5, $112-121$. http://dx.doi.org/10.4236/oja.2015.53010

[5] Brekhovskikh, L.M. (1973) Waves in Layered Media. Elsevier Science, Moscow.

[6] Flatte, S.M., Ed. (1982) Sound Transmission through a Fluctuating Ocean. Cambridge University Press, Moscow.

[7] Born, M.A. and Wolf, E. (1965) Principles of Optics.

[8] Landsderg, G.S. (1940) Optics.

\section{Submit or recommend next manuscript to SCIRP and we will provide best service for you:}

Accepting pre-submission inquiries through Email, Facebook, Linkedin, Twitter, etc A wide selection of journals (inclusive of 9 subjects, more than 200 journals)

Providing a 24-hour high-quality service

User-friendly online submission system

Fair and swift peer-review system

Efficient typesetting and proofreading procedure

Display of the result of downloads and visits, as well as the number of cited articles

Maximum dissemination of your research work

Submit your manuscript at: http://papersubmission.scirp.org/ 